\title{
Surgical strategy of bilateral synchronous sporadic renal cell carcinoma-experience of a Chinese university hospital
}

\author{
Xiao-Yi Hu${ }^{\dagger}$, Lei Xu ${ }^{\dagger}$, Jian-Ming Guo ${ }^{*}$ and Hang Wang ${ }^{*}$
}

\begin{abstract}
Background: The objective of this study is to investigate the optimal therapeutic protocol for BSSRCC.

Methods: A total of 32 BSSRCC patients, including 28 males and 4 females, were enrolled the study from January 2004 to May 2016. The diagnoses were defined by the measurements of CT, ultrasound, and MRI. Patients with hereditary syndrome were excluded. The management of surgical manner, operation staging, and sequence were scheduled in accordance with the tumor's location and size (based on Zhongshan score, ZS score), as well as the performance status of the patients. Among them, 8 cases were conducted with bilateral surgical procedure simultaneously and 24 cases were implemented with staged operations. NSS on the one side with contralateral RN, and NSS on both sides were performed in 17 and 15 patients separately.

Results: Thirty cases were conducted 56 operations in total. The average operation time was $260 \pm 52$ min in simultaneous operations and $162 \pm 40$ min in staged operations. The length of hospital stay in average was 11 . $5 \pm 1.8$ and $7.5 \pm 1.4$ days, respectively. Twenty-eight cases were followed up by $6-138$ months. The level of creatinine was elevated in 5 cases without hemodialysis conducted.

Conclusions: The location and size of the carcinomas, and the performance status of patients should be considered in determination of an appropriate surgical approach. Both renal function preservation and tumor eradication were similarly critical, whereas the latter is of more importance. ZS score may be helpful in the dilemma. Longer follow-up period and more patient enrolment are required.
\end{abstract}

Keywords: Kidney neoplasm, Nephron-sparing surgery, Radical nephrectomy, Synchronous, ZS score

\section{Background}

Bilateral synchronous sporadic renal cell carcinoma (BSSRCC) is an uncommon disease accounting for $3 \sim 4.2 \%$ renal carcinomas $[1,2]$. It is a difficult surgical dilemma for the urologist to formulate the therapeutic strategy for individual patient. Previous study disclosed patients with this type of tumor usually demonstrated a poor prognosis [3]. As usual, bilateral radical nephrectomy (RN) followed by renal replacement was the major management to these patients, which can obtain a $71 \%$ 5 -year survival in patients undergoing hemodialysis and

\footnotetext{
* Correspondence: guo.jianming@zs-hospital.sh.cn;

wang.hang@zs-hospital.sh.cn

${ }^{\dagger}$ Equal contributors

Department of Urology, Zhongshan Hospital, Fudan University, 180 Fenglin Rd, Shanghai 200032, China
}

86\% 5-year survival in patients receiving kidney transger beneficial ffect of cancer control for patients treated by bilateral RCC, with similar survival between synchronous and metachronous cancers. Meanwhile, the prognosis between the patients with NOMO synchronous bilateral RCC and the patients with NOMO unilateral RCC are comparable. Singer et al. [5] reported that the overall survival (OS) was $88 \%$ in the patients with synchronous sporadic carcinomas in a median 16-year follow-up period, which indicated the nephron-sparing surgery (NSS) was an essential procedure to bilateral renal tumors. They emphasized that all efforts should be of studies [5-7] suggested the favorable outcomes of the 
made during operation, so as to protect the renal function. In the present study, we summarize the experience in the treatment of the BSSRCC and suggest the selection criteria of the surgical manner.

\section{Methods}

Patients' characteristics

A total of 32 BSSRCC patients, including 28 males and 4 females at Zhongshan Hospital, Fudan University, were enrolled in the study between January 2004 and May 2016. The study was a prospective design, and the data were collected into an institutional review boardapproved database. The patients with bilateral metachronous renal tumors, cystic renal masses, familial renal cell carcinoma syndromes, urothelial cell carcinomas, and defined hereditary syndromes were excluded in accordance with the diagnosis criteria [8]. ECOG PS of all the patients were $0 \sim 2$.

Among all patients, 22 cases were clued unexpectedly in an annual routine physical examination, 5 cases were diagnosed with major complaint of flank pain in clinic and 5 cases were defined due to hematuria. All diagnoses were established depending on the evidence of bilateral renal masses on ultrasound and CT imagings before surgery. Intravenous urography (IVU) was adopted in 12 cases, in which 10 were identified with the compression of unilateral or bilateral renal pelvis or calyx. MRI demonstrated the bilateral renal masses in all 14 patients. Cancer embolus in the vena cava was detected in one patient and megatherium lymph nodes in the retroperitoneum were found in another patient. None of patients showed the signs of distant metastasis. According to the 2009 UICC/AJCC TNM staging system, 44 (68.8\%) tumors were classified at T1aN0M0, $13(20.3 \%)$ tumors were at T1bN0M0, 5 (7.8\%) tumors were at T2N0M0, 1 (1.6\%) tumor was at T3bN0M0, and other 1 (1.6\%) tumor was at T2N1M0 (Table 1).

All tumors were stratified into three complexity levels according to Zhongshan score (ZS score) [9]. Low-risk tumors were scored between 3 4, whereas moderate tumors were scored between $5 \sim 7$, and high-risk tumors were scored $\geq 8$.

\section{Preoperative work-up}

The preoperative work-up consists of medical history, physical examinations, and routine laboratory tests. Concentrations of serum creatinine were recorded before and after operation consecutively at an interval of 36 months. Estimated Glomerular filtration rate (GFR) was calculated using the modification of diet in renal disease (MDRD) formula. Postoperative assessments during follow-up period included abdominal ultrasonography and chest $\mathrm{x}$-ray examinations at the interval of
Table 1 Baseline demographic and clinical characteristics of the BSSRCC patients

\begin{tabular}{|c|c|c|}
\hline Characteristics & \multicolumn{2}{|c|}{ Number(percentage), $N=32$} \\
\hline \multicolumn{3}{|l|}{ Gender } \\
\hline Male & \multicolumn{2}{|l|}{$28(87.5 \%)$} \\
\hline Female & \multicolumn{2}{|l|}{$4(12.5 \%)$} \\
\hline \multicolumn{3}{|l|}{ Age (years) } \\
\hline$\geq 50$ & \multicolumn{2}{|l|}{$21(65.6 \%)$} \\
\hline$<50$ & \multicolumn{2}{|l|}{$11(34.4 \%)$} \\
\hline \multicolumn{3}{|l|}{ Operation stage } \\
\hline One-stage & \multicolumn{2}{|l|}{$8(25.0 \%)$} \\
\hline Two-stage & \multicolumn{2}{|l|}{$24(75.0 \%)$} \\
\hline TNM stage & Right side, $N=32$ & Left side, $N=32$ \\
\hline $\mathrm{T}_{1 \mathrm{a}} \mathrm{N}_{0} \mathrm{M}_{0}$ & $21(65.6 \%)$ & $23(71.8 \%)$ \\
\hline $\mathrm{T}_{1 \mathrm{~b}} \mathrm{~N}_{0} \mathrm{M}_{0}$ & $9(28.1 \%)$ & $4(12.5 \%)$ \\
\hline $\mathrm{T}_{2 \mathrm{a}} \mathrm{N}_{0} \mathrm{M}_{0}$ & $1(3.1 \%)$ & $1(3.1 \%)$ \\
\hline $\mathrm{T}_{2 b} \mathrm{~N}_{0} \mathrm{M}_{0}$ & 0 & $3(9.4 \%)$ \\
\hline $\mathrm{T}_{3 b} \mathrm{~N}_{0} \mathrm{M}_{0}$ & $1(3.1 \%)$ & 0 \\
\hline $\mathrm{T}_{2} \mathrm{~N}_{1} \mathrm{M}_{0}$ & 0 & $1(3.1 \%)$ \\
\hline Operation Sequence & $N=32$ & $N=32$ \\
\hline First & $12(37.5 \%)$ & $20(62.5 \%)$ \\
\hline Second & $20(62.5 \%)$ & $12(37.5 \%)$ \\
\hline Operation methods & $N=32$ & $N=32$ \\
\hline NSS & $24(75.0 \%)$ & $23(71.9 \%)$ \\
\hline $\mathrm{RN}$ & $8(25.0 \%)$ & $9(28.1 \%)$ \\
\hline ZS score & $N=24$ & $N=23$ \\
\hline Low risk & $5(20.8 \%)$ & $6(26.1 \%)$ \\
\hline Moderate & $10(41.6 \%)$ & $8(33.3 \%)$ \\
\hline High risk & 9 (37.5\%) & 9 (39.1\%) \\
\hline
\end{tabular}

each 3 months in the first 2 years and at each 6 months afterward.

\section{Treatment}

One-stage bilateral surgery was performed in eight patients, of which six cases were carried out bilateral NSS and two cases were taken RN for one side first and NSS for the opposite side thereafter. Two-stage operations were performed in other 24 cases, of which 5 cases were adopted NSS first and RN for the opposite side 4-8 weeks later; 10 cases were performed RN first and then NSS for the opposite side 4-8 weeks later; 9 cases were conducted NSS in both sides within 4-8 weeks. For one patient with tumor embolus in vena cava, an intravenous stent was placed and a filter was positioned at inferior vena cava before operation to prevent the embolus occlusion, followed by ipsilateral RN and contralateral NSS 


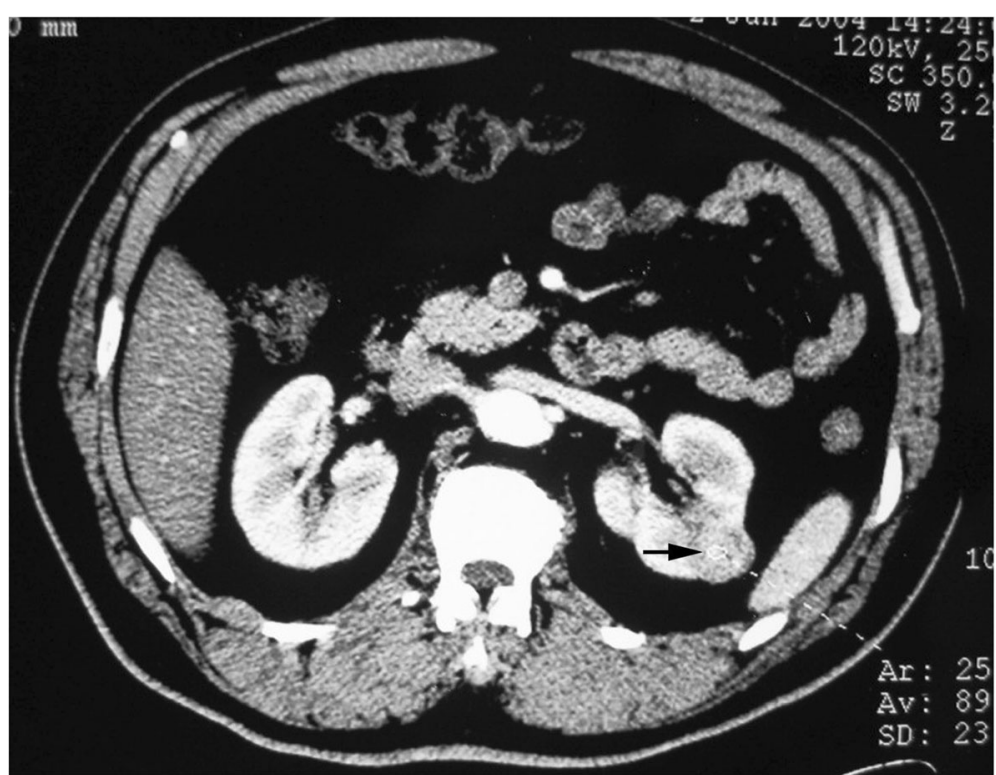

Fig. 1 Bilateral NSS: larger tumor on the left side was dissected first

7 weeks later. The characteristics of tumor size and the therapeutic options are summarized in Table 1. Complications were evaluated using the Clavien scale [10].

\section{Surgical strategy for BSSRCC}

\section{Selection of surgical stage}

One-stage bilateral surgery (OBS): OBS is considered when physical status of the patient is fitted $(E C O G$ PS $=0$ ) while being without any comorbidity.
Two stages surgery (TSS): TSS is selected for the patient with large size tumor or other complications, such as cancer embolus in the vena cava. Patients with ECOG PS $>0$ are also enrolled in this group.

\section{Surgical sequence}

For BSSRCC patients intending staged surgery, the choosing of side to be operated initially and the surgical approach should be meticulously decided based on the

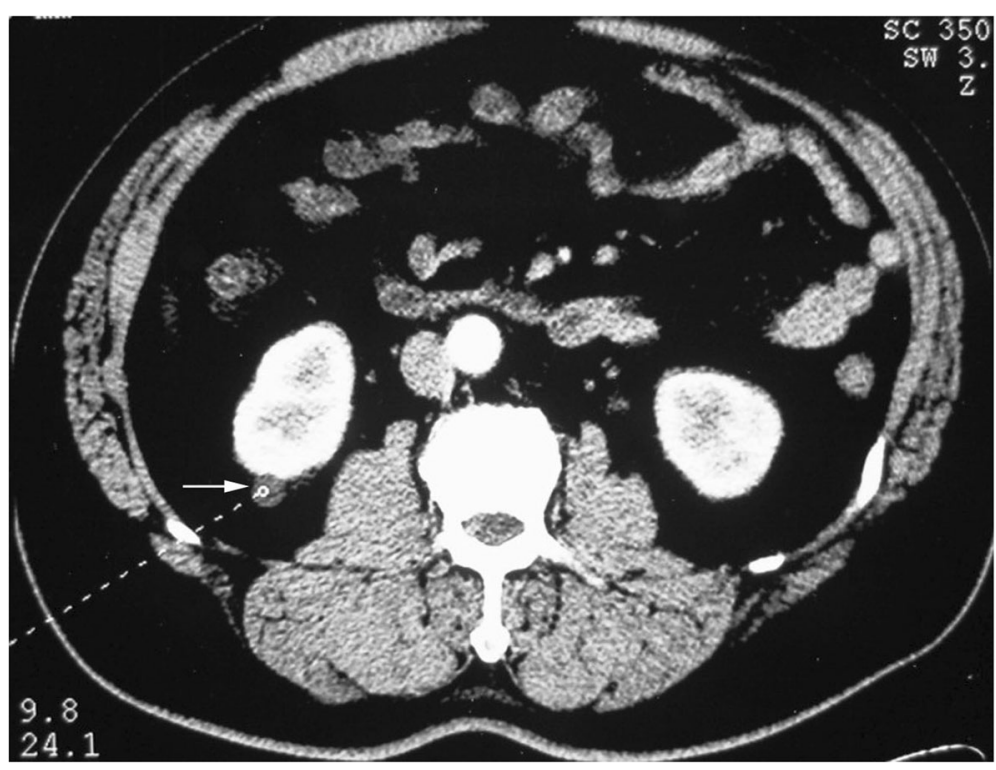

Fig. 2 Bilateral NSS: larger tumor on the left side was dissected first (Figs. 1 and 2 were of the same patient) 


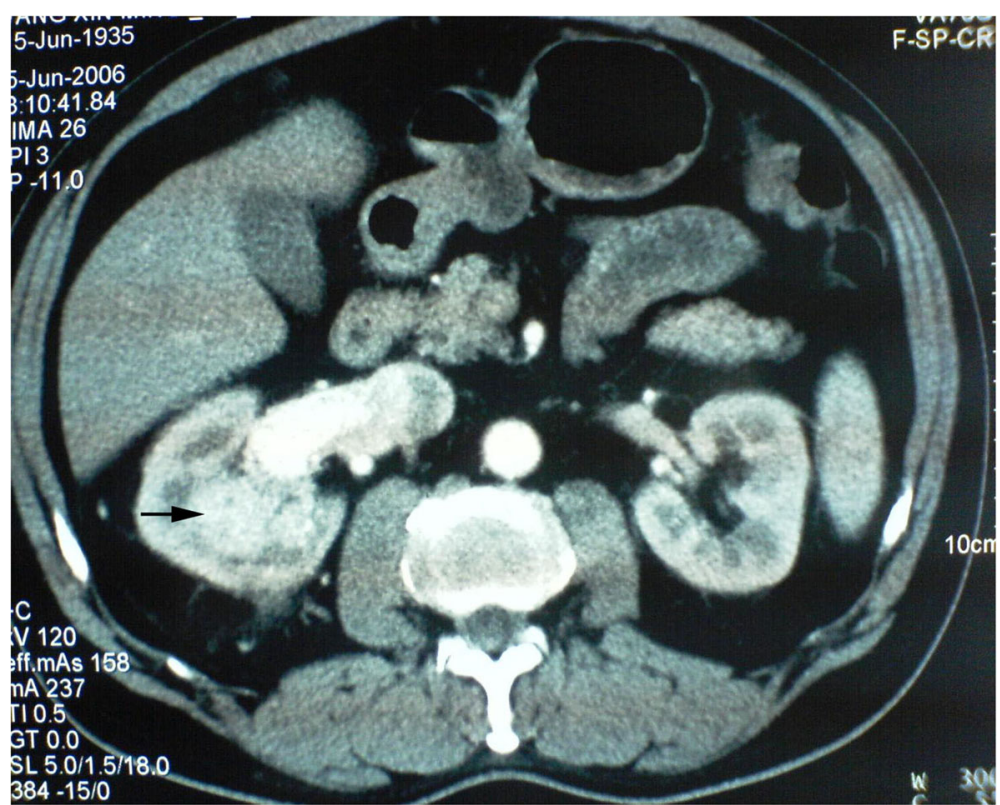

Fig. 3 RN on the right side first and NSS on the left thereafter at second staged surgery

tumor location and size at both the kidneys. For the bilateral NSS, first-stage surgery should be conducted on the side with higher ZS score tumors (Figs. 1 and 2). For the patients determined to do radical and partial resection separately at both sides one after another, the first-stage radical surgery should be operated on the side with larger tumor as well, if the partial resection to low-moderate risk tumors in the other side were easy to perform expectedly
(Figs. 3 and 4). However, if tumors were assessed at high-risk level on the one side which were planned to do partial resection, the NSS procedure should be operated earlier than contralateral RN (Fig. 5). If NSS was unsuccessful and required to switch to RN, further preparations including appropriate hemodialysis were necessary prior to the second-stage operation of contralateral RN (Table 2).

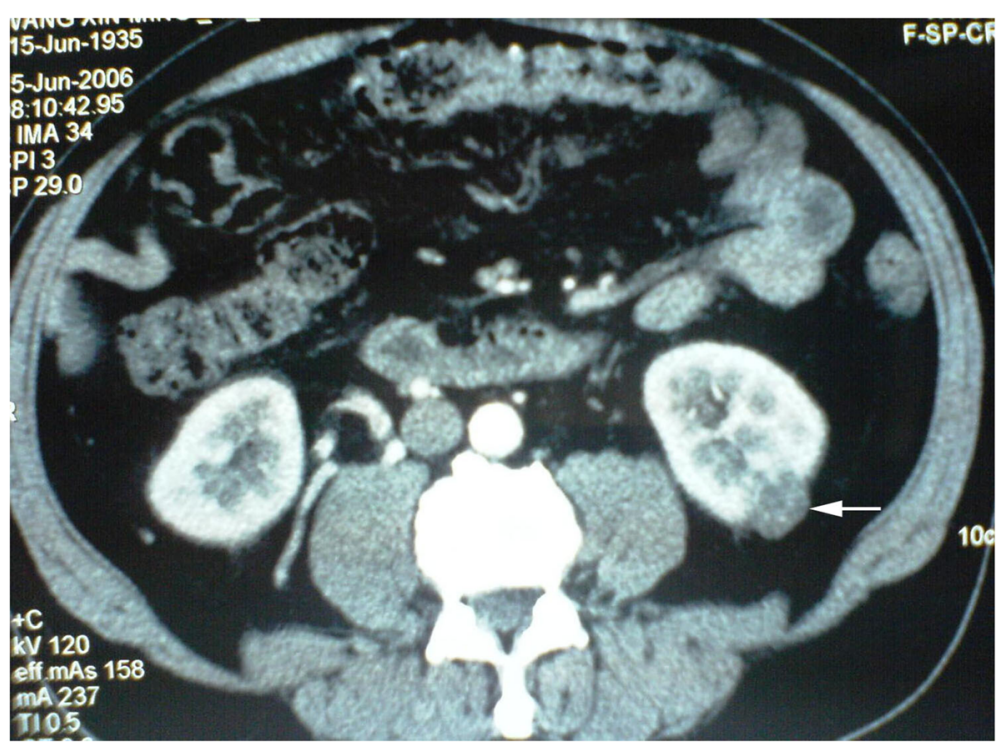

Fig. 4 RN on the right side first and NSS on the left thereafter at second staged surgery (Figs. 3 and 4 were of the same patient) 


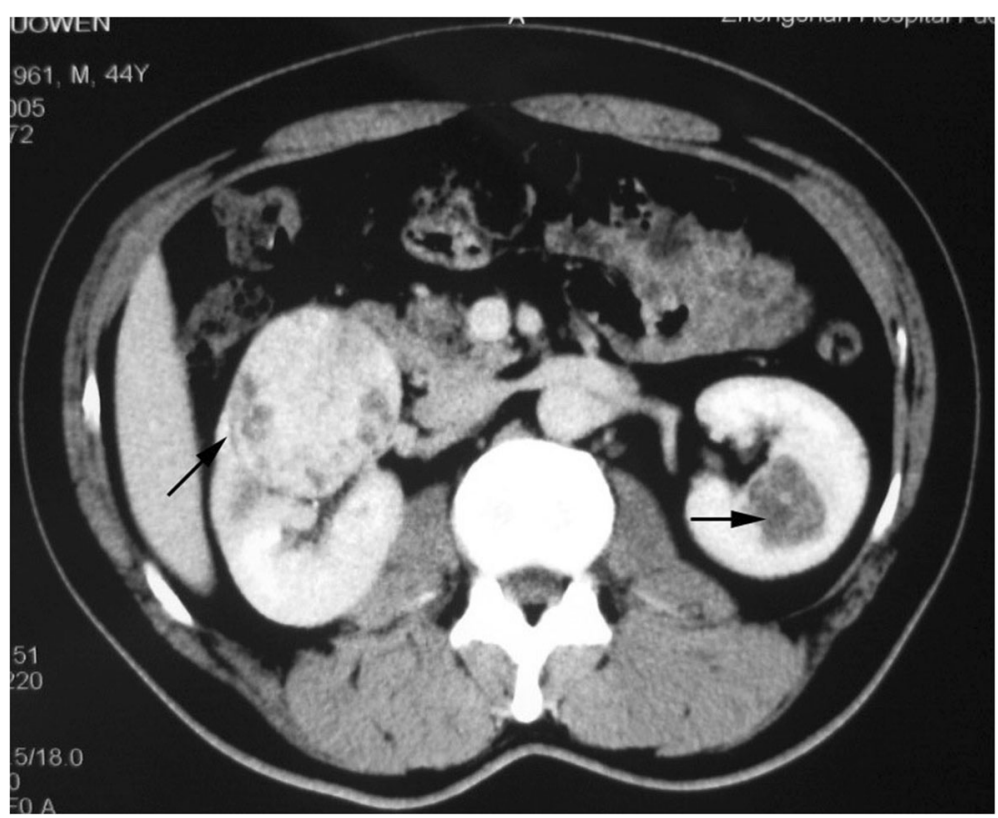

Fig. 5 NSS on the left side first and RN on the right side at second staged surgey

The same criteria should be met in one-stage bilateral surgery in management of surgical schedule.

\section{Results}

A total of 56 operations were productively completed in all 32 patients (average age 54.7 years, range 31 to 77 ). Surgical patterns of retroperitoneal open partial nephrectomy (OPN) and transperitoneal open/laparoscopic radical nephrectomy were selectively applied. Mean operation time of OBS and TSS were $263 \pm 50$ min (range 200-320) and $154 \pm 42 \mathrm{~min}$ (range105-180), mean hospitalization time of OBS and TSS were 11.4 \pm 1.9 days (range 8-15) and $7.4 \pm 1.3$ days (range $6-9$ ), respectively. The warm ischemia time in NSS was $26 \pm 7$ min (range 16-45). Pathological examination of 64 neoplasms samples revealed that 57 samples were clear cell carcinoma, 5 were papillary carcinoma, and 2 were chromophobe carcinoma.

After bilateral NSS, one patient appeared pseudoaneurysm of renal arteriole, accompanied with gross hematuria and hypovolemic shock. The patient then received the treatment of percutaneous high-selective renal arterial embolization (Clavien grade 3). Twenty-eight patients were followed up 6-138 months (median 89 months) except four

Table 2 Surgical sequence of the BSSRCC based on ZS score

\begin{tabular}{lll}
\hline & First step & Second step \\
\hline Bilateral NSS & Higher risk side & Lower risk side \\
RN+NSS & RN & NSS (low-moderate risk) \\
& NSS (high risk) & RN \\
\hline
\end{tabular}

patients were lost contact. The renal function was maintained in normal range in 23 patients. Increased creatinine $(125-187 \mu \mathrm{mol} / \mathrm{L})$ level and reduced eGFR were happened in five patients, who were treated with NSS on the one side and contralateral $\mathrm{RN}$ without hemodialysis. Lung metastasis was found in one patient after 14 months of RN plus contralateral NSS operation and died 25 months later. Tumor recurrence at the left nephridial pit was seen in one patient conducted with NSS on the both sides. The patient was treated with salvage radiotherapy and died 38 months later after surgery. One patient with RN and contralateral NSS died from cerebrovascular accident 6 months after surgery (Table 3).

\section{Discussion}

Although BSSRCC is an uncommon disease with lower incidence [11], the management is much more difficult

Table 3 Results of following up of the BSSRCC patients

\begin{tabular}{ll}
\hline Characteristics & Number(percentage) \\
\hline Lost in follow-up & $4(12.5 \%)$ \\
Complications & \\
$\quad$ Clavien I II & $5(17.9 \%)$ \\
Clavien III & $1(3.6 \%)$ \\
CRI & $5(17.9 \%)$ \\
Hemodialysis & 0 \\
Survival & $25(89.3 \%)$ \\
Metastasis/recurrence & $1 / 1(3.6 \% / 3.6 \%)$ \\
\hline
\end{tabular}


and complicated than that of metachronous RCC. Accordingly, the selection of surgical approach and the schedule of operation sequence require careful evaluation of the disease and the general condition of patients.

\section{Surgical criteria}

\section{Selection of operation pattern in our study}

1). Bilateral NSS: The location and size of the tumor in both sides meet the indication for NSS (Fig. 6). In present series, 15 patients were implemented bilateral NSS.

2). Lateral NSS on the one side and RN on the contralateral side. In general, it was applied for patients with small tumor on the one side which meet the indication for NSS and with relatively larger tumor on the other side which required RN. Seventeen patients in the cohort received surgery in this manner (Fig. 7). Four patients showed the elevation of creatinine levels after operation because of inadequate preservation of renal parenchyma. It indicated the renal parenchyma should be conserved as much as possible in NSS to avoid postoperative dialysis.

3). Bilateral RN. It is applied in patients with bilateral large tumors which are unsuitable for NSS. Due to the complete loss of renal function after surgery, kidney replacements are necessary to maintain the life of patients. Therefore, the selection of this surgical manner should be particularly cautious. None of our patient has taken this surgical manner.

\section{Surgery sequence}

The individual therapeutic protocol for each patient should be created only through appropriately weighing the advantages and disadvantages of different surgical sequences, then make the selection of either one-stage or two-stage surgery manner and which side is the first for surgery. The essential principle is which manner can provide the optimal effects of thoroughly eliminating the tumor cells as early as possible. One-stage bilateral surgery may be the effective therapeutic method for BSSRCC based on the criteria in case of physical status of the patient allowed. However, the elderly patients or those with vital organ dysfunction owing to complicated status of operation either in one or two sides is better to take staged surgery after 1-2 months of the first-stage surgery done. The initial operation on one side is a pivotal procedure in staged surgery. We should focus on the high risk tumor of BSSRCC following the above criteria. But it is not always the case. Maximal preservation of renal function should be taken into account at the same time, especially in the case of NSS with contralateral RN. Consequently, we describe the therapeutic methods in combination of renal function preservation and tumor removal in making our surgical criteria.

In summary, both preservation of the renal parenchyma and tumor eradication should be considered simultaneously in management of BSSRCC. Before formulating an individual therapeutic regime, the patient's performance status, tumor size, and location should be evaluated carefully [12] according to the ZS score. The renal parenchyma should be preserved as much as possible during the operation to prevent

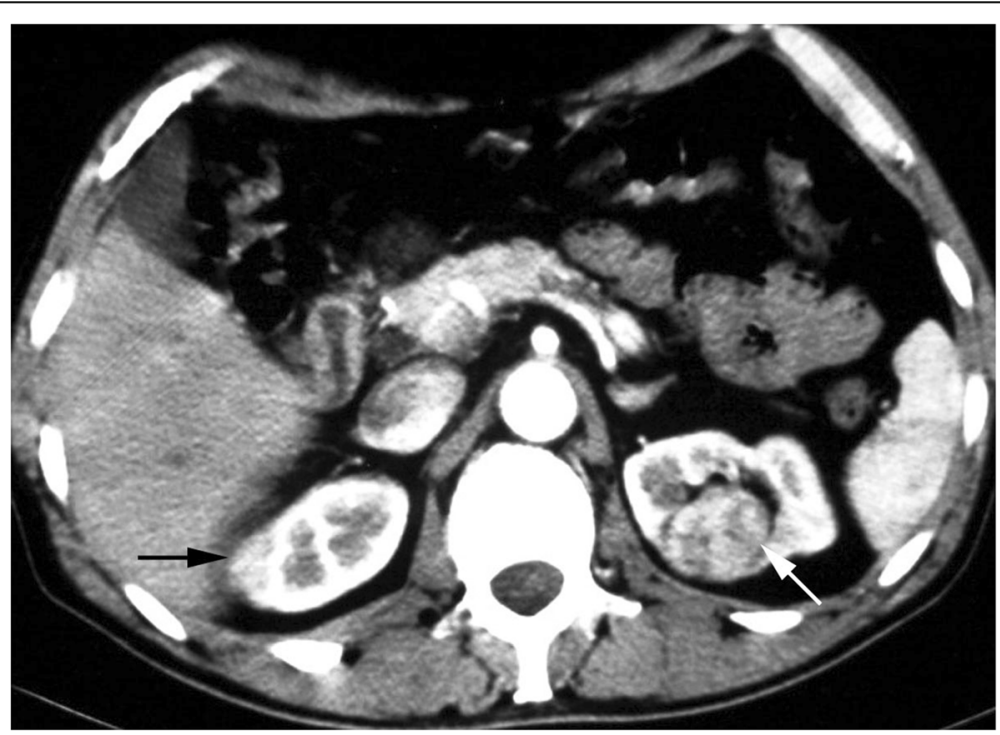

Fig. 6 Left central renal tumor was partially resected 


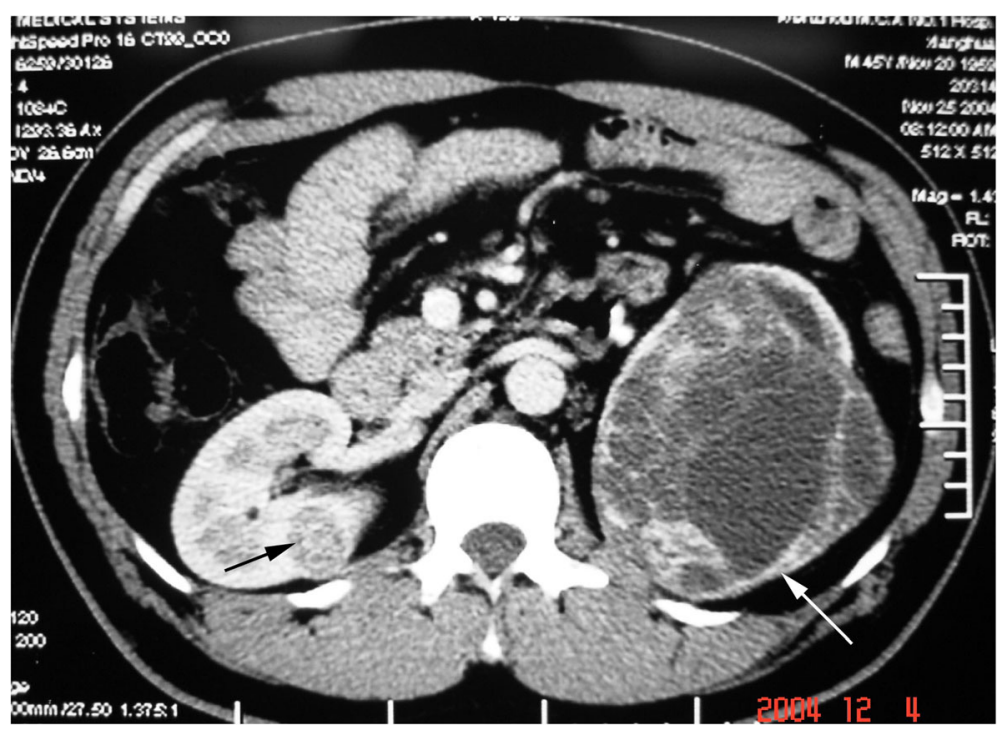

Fig. 7 NSS on the right side and RN on the left

hemodialysis postoperatively, but complete tumor removal is the most important target for the treatment of patient.

Our newly established surgical criteria for BSSRCC, using our novel and simple score system, have demonstrated encouraging and satisfactory outcomes (Fig. 8). It is feasible and safe in clinical application. Since BSSRCC is a rare disease $[13,14]$, the longer follow- up period, more patient enrolment, and novel anatomic classification systems [15] are needed to further investigate the beneficial effects of different surgical approaches for the patients.

\section{Conclusions}

We established our promising surgical criteria for BSSRCC based on the ZS score.

Both preservation of renal parenchyma and tumor eradication should be considered in BSSRCC. Before formulating a therapeutic regime, the patient's performance status, tumor size, and location should be evaluated. Renal parenchyma should be preserved as much as possible during the operation, but complete tumor removal is more important.

Longer follow-up and more patients enrolment are required.

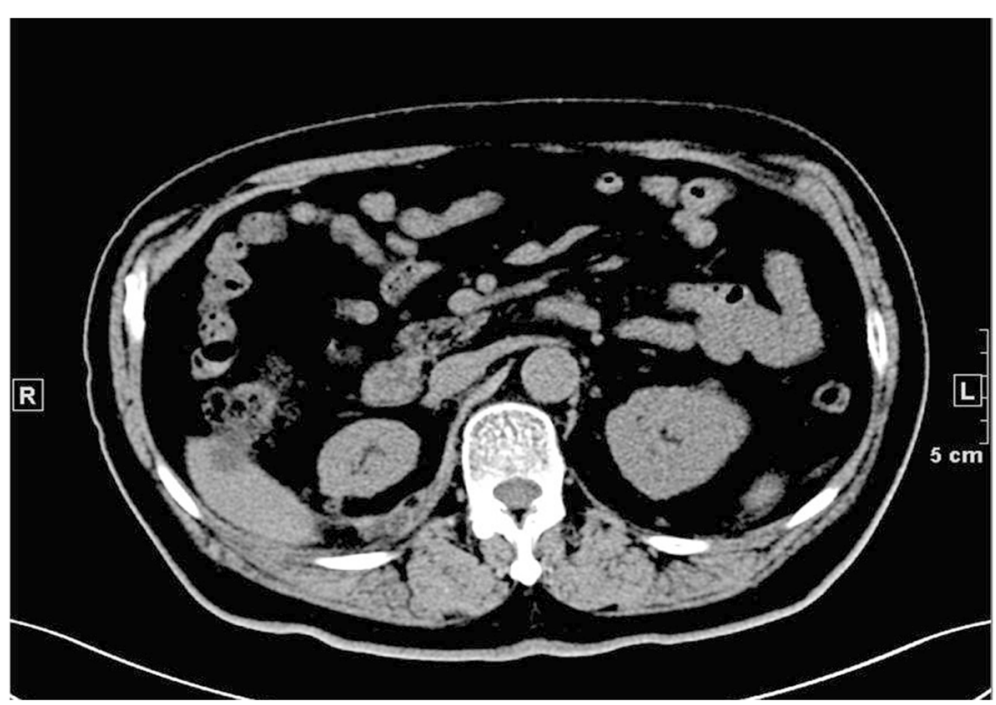

Fig. 8 One year after bilateral NSS with normal renal function and no tumor recurrence could be found 


\section{Abbreviations}

BSSRCC: Bilateral synchronous sporadic renal cell carcinoma; CRI: Chronic renal insufficiency; ECOG PS: Eastern cooperative oncology group, performance status; GFR: Glomerular filtration rate; IVU: Intravenous urography; NSS: Nephron-sparing surgery; OBS: One-stage bilateral surgery; OPN: Open partial nephrectomy; OS: Overall survival; RN: Radical nephrectomy; TSS: Two stages surgery; ZS score: Zhongshan score

\section{Acknowledgements}

The authors thank Ms. Shicui Liu of China Oncology Medical Affairs of Pfizer Investment Company Ltd. for the english check and sentence polishing.

\section{Funding}

Not applicable.

\section{Availability of data and materials}

Data sharing is not applicable to this article as no datasets were generated or analysed during the current study.

\section{Authors' contributions}

XYH and LX carried out the main studies and drafted the manuscript. JMG and HW participated in the research design and coordination while HW performed all the operations. All authors read and approved the final manuscript.

\section{Competing interests}

The authors declare that they have no competing interests.

\section{Consent for publication}

We have obtained consent to publish from all the patients to report their individual data in this article.

\section{Ethics approval and consent to participate}

This study has been approved by the ethics committee of Fudan University and has therefore been performed in accordance with the ethical standards laid down in the Declaration of Helsinki 1964 and its later amendment.

\section{Received: 9 March 2016 Accepted: 15 December 2016}

\section{Published online: 28 February 2017}

\section{References}

1. Grimaldi G, Reuter V, Russo P. Bilateral non-familial renal cell carcinoma. Ann Surg Oncol. 1998;5(6):548-52

2. Siegel R, Naishadham D, Jemal A. Cancer statistics, 2012. CA Cancer J Clin. 2012:62(1):10-29.

3. Jacobs SC, Berg Sl, Lawson RK. Synchronous bilateral renal cell carcinoma: total surgical excision. Cancer. 1980;46:2341-5.

4. Black J, Rotellar C, Rakowski TA, et al. Bilateral nephrectomy and dialysis as an option for patients with bilateral renal cancer. Nephron. 1988:49:150-3.

5. Singer EA, Vourganti S, Lin KY, et al. Outcomes of patients with surgically treated bilateral renal masses and a minimum of 10 years of follow-up. J Urol. 2012;188(6):2084-8.

6. Klatte T, Wunderlich H, Patard JJ, et al. Clinicopathological features and prognosis of synchronous bilateral renal cell carcinoma: an international multicentre experience. BJU Int. 2007;100(1):21-5.

7. Becker F, Siemer S, Tzavaras A, et al. Long-term survival in bilateral renal cell carcinoma: a retrospective single-institutional analysis of 101 patients after surgical treatment. Urology. 2008;72(2):349-53.

8. Campbell SC, Lane BR. Campbell-Walsh Urology (11th Edition), vol. chapter 57. 2016. p. 1320-41

9. Zhou L, Guo J, Wang H, Wang G. The Zhongshan score: a novel and simple anatomic classification system to predict perioperative outcomes of nephron-sparing surgery. Medicine (Baltimore). 2015;94(5), e506.

10. Mitropoulos D, Artibani W, Graefen M, et al. Reporting and grading of complications after urologic surgical procedures: an ad hoc EAU guidelines panel assessment and recommendations. Eur Urol. 2012;61:341-9.

11. Mu DW, Guo T, Zhang CJ, et al. Prognostic and long term follow-up analysis of sporadic bilateral renal cell carcinoma. Beijing Da Xue Xue Bao. 2013; 45(4):562-6

12. Ching CB, Li J, Gill IS, et al. Functional and oncologic outcomes of bilateral open partial nephrectomy versus bilateral laparoscopic partial nephrectomy. J Endourol. 2011;25(7):1193-7.
13. Woodson B, Fernandez R, Stewart C, et al. Bilateral synchronous sporadic renal masses: intermediate functional and oncological outcomes at a single institution. Int Urol Nephrol. 2013;45(3):619-25.

14. Karthikeyan VS, Dorairajan LN, Kumar S, et al. Sporadic bilateral synchronous multicentric papillary renal cell carcinoma masquerading as bilateral multifocal pyelonephritis. Ann R Coll Surg Engl. 2014;96(5):e7-10.

15. Zhou L, Cao Y, Bian T, et al. Number of renal columns invaded by tumor: a novel parameter for predicting complexity and outcomes of off- clamp open partial nephrectomy. J Am Coll Surg. 2015;221(2):539-49.

\section{Submit your next manuscript to BioMed Central and we will help you at every step:}

- We accept pre-submission inquiries

- Our selector tool helps you to find the most relevant journal

- We provide round the clock customer support

- Convenient online submission

- Thorough peer review

- Inclusion in PubMed and all major indexing services

- Maximum visibility for your research

Submit your manuscript at www.biomedcentral.com/submit
) BioMed Central 